\title{
Allylic Oxidation of Ester-substituted 1,4-Dienes
}

\section{Supporting Information}

\author{
Lars E. Sattler ${ }^{\mathrm{a}, \mathrm{b}}$ and Gerhard Hilt $\mathrm{t}^{\mathrm{b}}$
}

${ }^{a}$ Fachbereich Chemie, Philipps-Universität Marburg, Hans-Meerwein-Str. 4, 35043 Marburg

${ }^{\mathrm{b}}$ Institut für Chemie, Carl von Ossietzky Universität Oldenburg, Carl-von-Ossietzky-Str. 9-11, 26129 Oldenburg

* gerhard.hilt@uni-oldenburg.de

\section{Table of Contents}

${ }^{1 \mathrm{H}}$ and $\left.{ }^{13 \mathrm{C}_{\{}}{ }^{1 \mathrm{H}}\right\}$ NMR spectra of compounds $\quad$ S2

2,3-Dimethyl-5-methyleneundec-2-ene (1a) S2

(E)-tert-Butyl((5,6-dimethylhepta-2,5-dien-1-yl)oxy)dimethylsilane (1c) S3

(Z)-Trimethyl((2-methylenehex-4-en-1-yl)oxy)silane (1d) S4

Ethyl (2Z,5Z)-hepta-2,5-dienoate (1f-Z) S5

Ethyl (2E,5Z)-hepta-2,5-dienoate (1f-E) S6

$\begin{array}{ll}\text { Ethyl (2E,5Z)-5-methylhepta-2,5-dienoate (1g) S7 } & \text { S }\end{array}$

Ethyl (2E,4E)-6-hydroxy-5,6-dimethylhepta-2,4-dienoate (4e) S8

Ethyl $(2 E, 4 E)$-6-hydroxyhepta-2,4-dienoate $(\mathbf{4 f}) \quad$ S9

Ethyl (2E,4E)-6-hydroxy-5-methylhepta-2,4-dienoate (4g) S10

Ethyl (2E,4E)-6-methoxy-5,6-dimethylhepta-2,4-dienoate (5) S11

Ethyl (2E,4E)-6-ethoxy-5,6-dimethylhepta-2,4-dienoate (6) S12

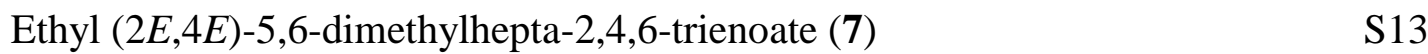

$\begin{array}{ll}\text { Ethyl }(2 E, 4 E)-6-m e t h o x y h e p t a-2,4-d i e n o a t e ~(8) & \text { S14 }\end{array}$

Ethyl (2E,5Z)-5-methyl-4-oxohepta-2,5-dienoate (9) S16

Ethyl (2E,4E)-6-methoxy-5-methylhepta-2,4-dienoate (10) S17

Ethyl (2E,5E)-3-methyl-4-oxo-6-(trimethylsilyl)hexa-2,5-dienoate (11) S18

${ }^{77 \mathrm{Se}}$ NMR spectra of $\mathrm{SeO} 2$ in CD3CN/D2O 1:1 (v/v) S19

GC-FID and GC/MS spectra for entry 3 in Table 1 (reaction optimization) S20

$\begin{array}{ll}\text { References } & \text { S20 }\end{array}$ 
${ }^{1} \mathrm{H}$ and ${ }^{13} \mathrm{C}\left\{{ }^{1} \mathrm{H}\right\}$ NMR spectra of compounds

2,3-Dimethyl-5-methyleneundec-2-ene (1a)

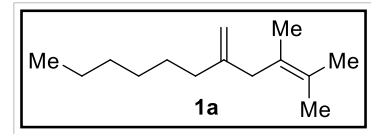

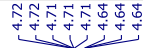


(E)-tert-Butyl((5,6-dimethylhepta-2,5-dien-1-yl)oxy)dimethylsilane (1c) $\mathrm{tBuMe}_{2} \mathrm{SiO}_{1 \mathrm{Me}}^{\mathrm{Me}} \mathrm{Me}$

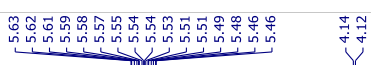
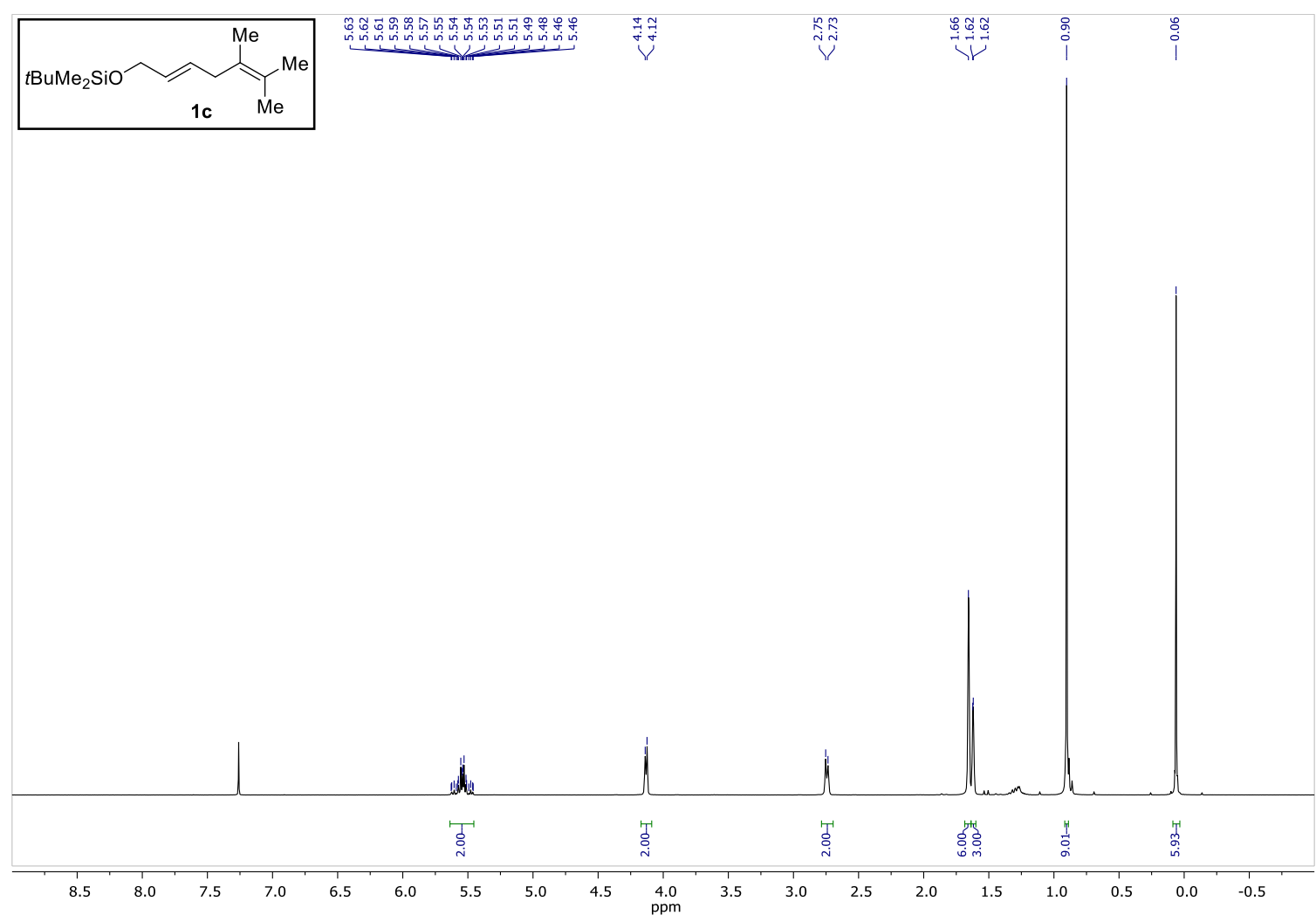

${ }^{1} \mathrm{H}$ NMR (300 MHz CDCl 3 )
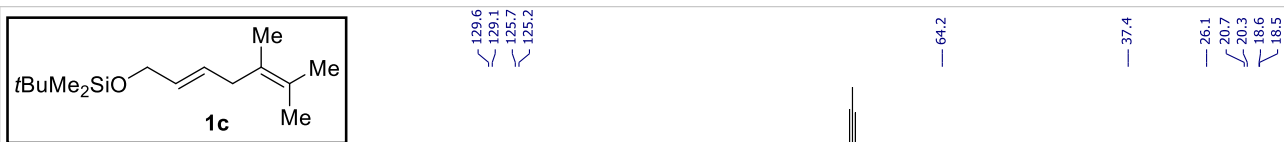

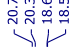
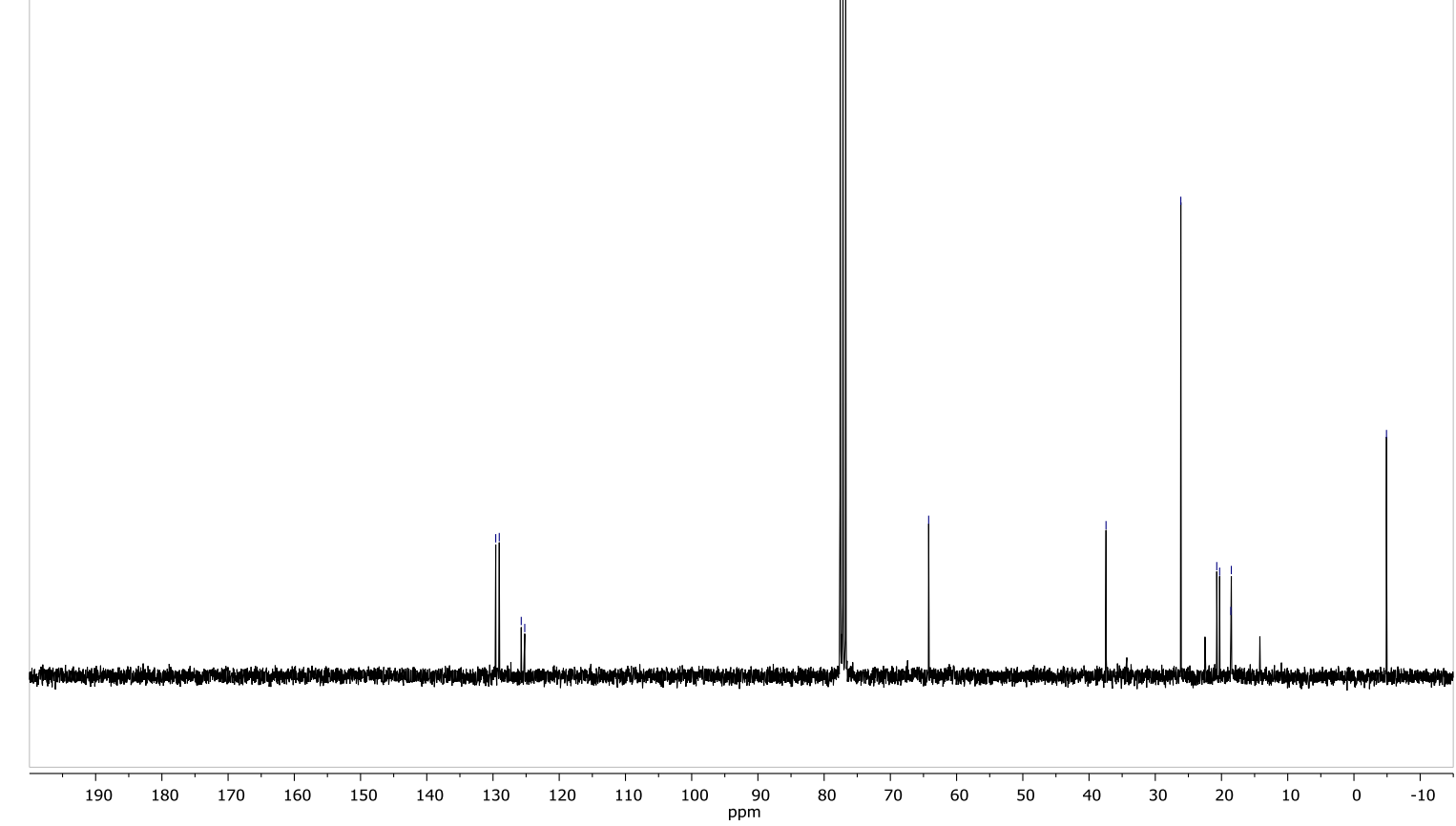

${ }^{13} \mathrm{C}\left\{{ }^{1} \mathrm{H}\right\} \mathrm{NMR}\left(75 \mathrm{MHz}, \mathrm{CDCl}_{3}\right)$ 
(Z)-Trimethyl((2-methylenehex-4-en-1-yl)oxy)silane (1d)

$\mathrm{Me}_{3} \mathrm{SiO} \mathrm{Id}_{\mathrm{Me}}$

ไู่
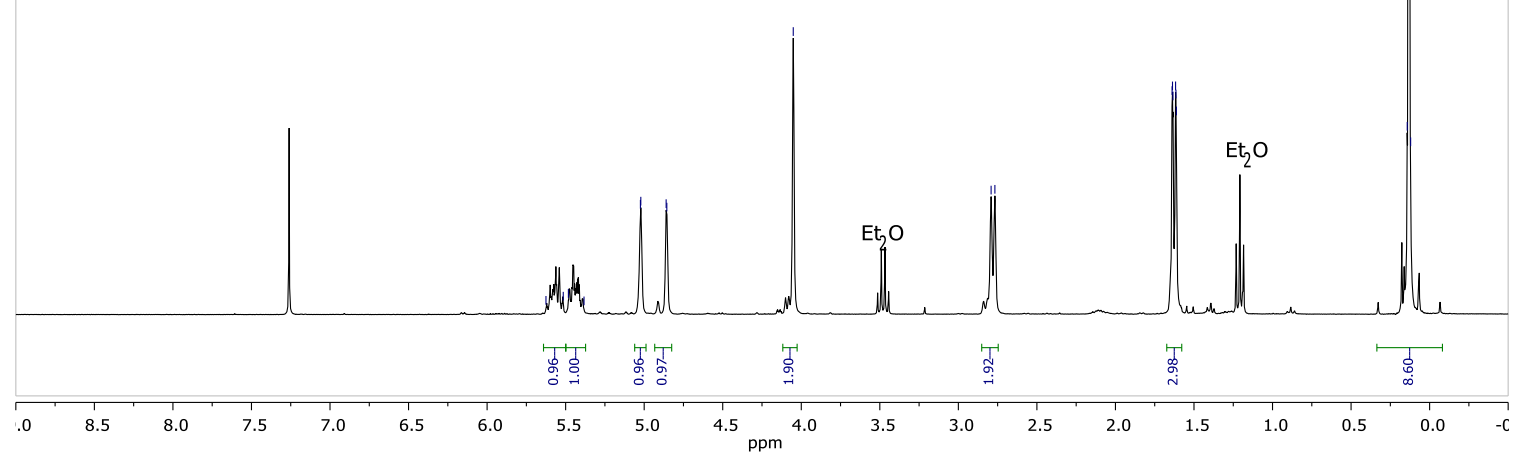

${ }^{1} \mathrm{H}$ NMR (300 MHz $\mathrm{CDCl}_{3}$ )
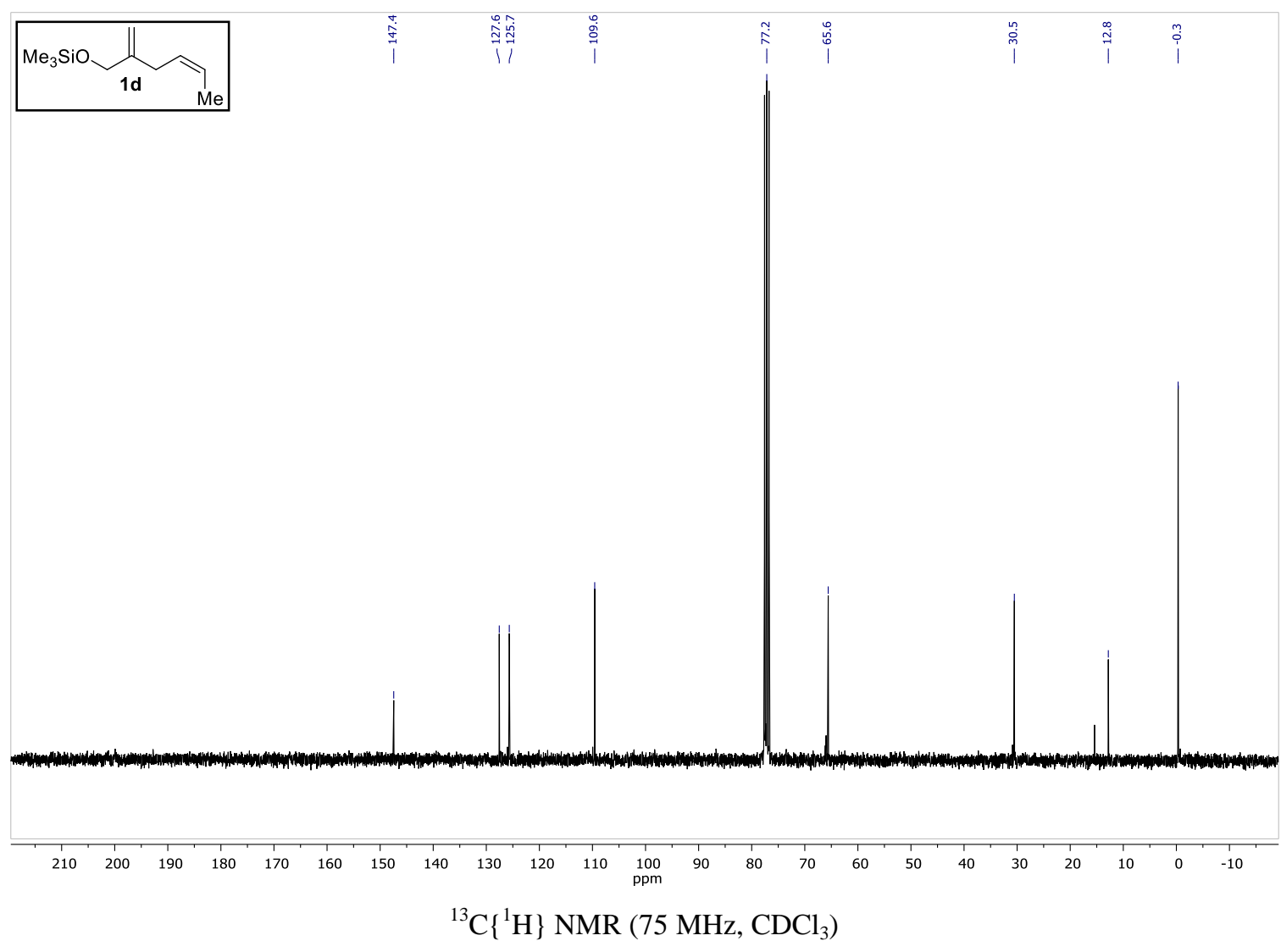
Ethyl (2Z,5Z)-hepta-2,5-dienoate (1f-Z)
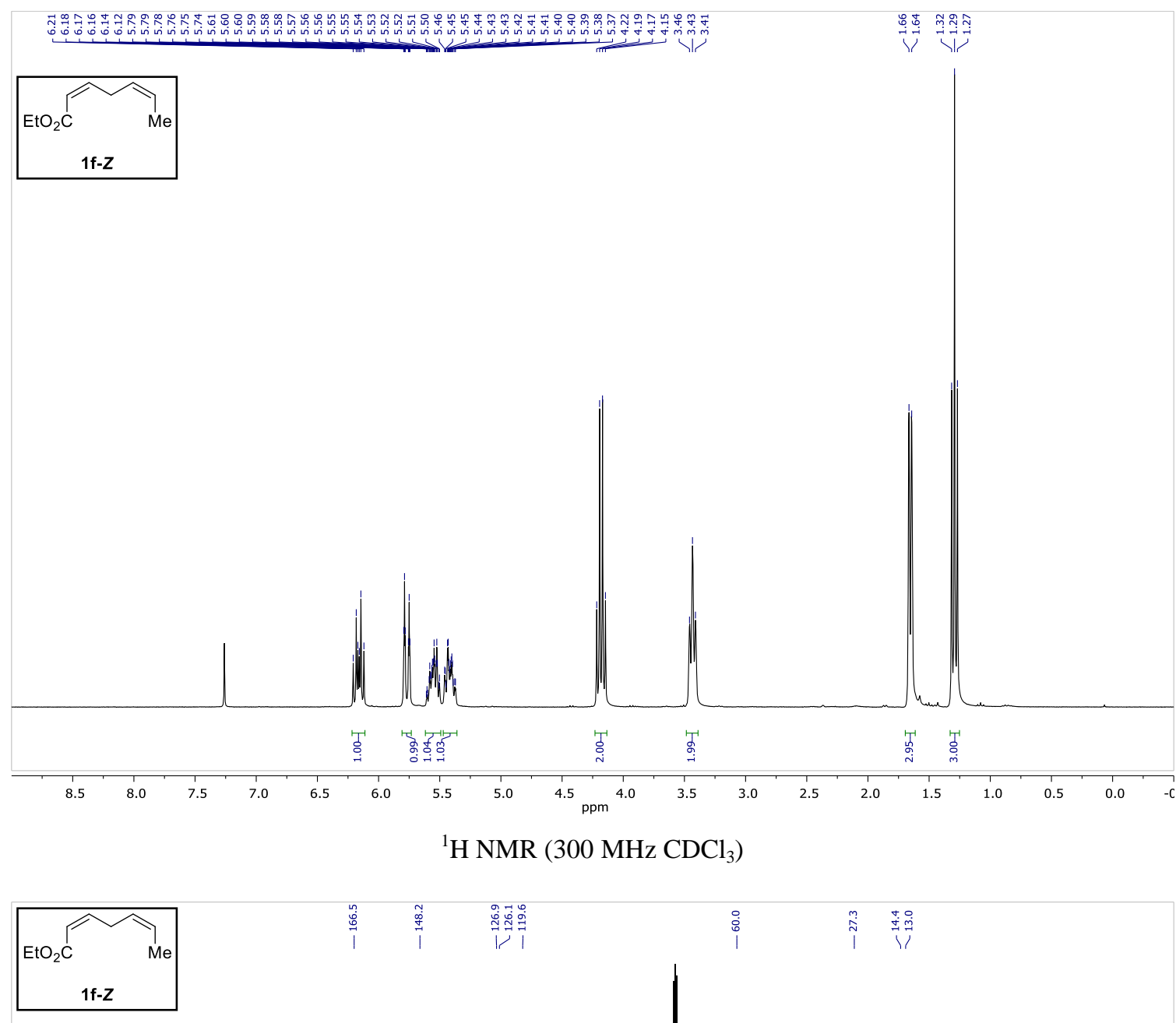

$\begin{array}{lllllllllllllllllllllllllllllllllllllllll} & 260 & 250 & 240 & 230 & 220 & 210 & 200 & 190 & 180 & 170 & 160 & 150 & 140 & 130 & 120 & 110 & 100 & 90 & 80 & 70 & 60 & 50 & 40 & 30 & 20 & 10 & 0 & -10 & -20 & -30 & -40 & -50 & -6\end{array}$

${ }^{13} \mathrm{C}\left\{{ }^{1} \mathrm{H}\right\}$ NMR (75 MHz, $\left.\mathrm{CDCl}_{3}\right)$ 
Ethyl (2E,5Z)-hepta-2,5-dienoate (1f-E)
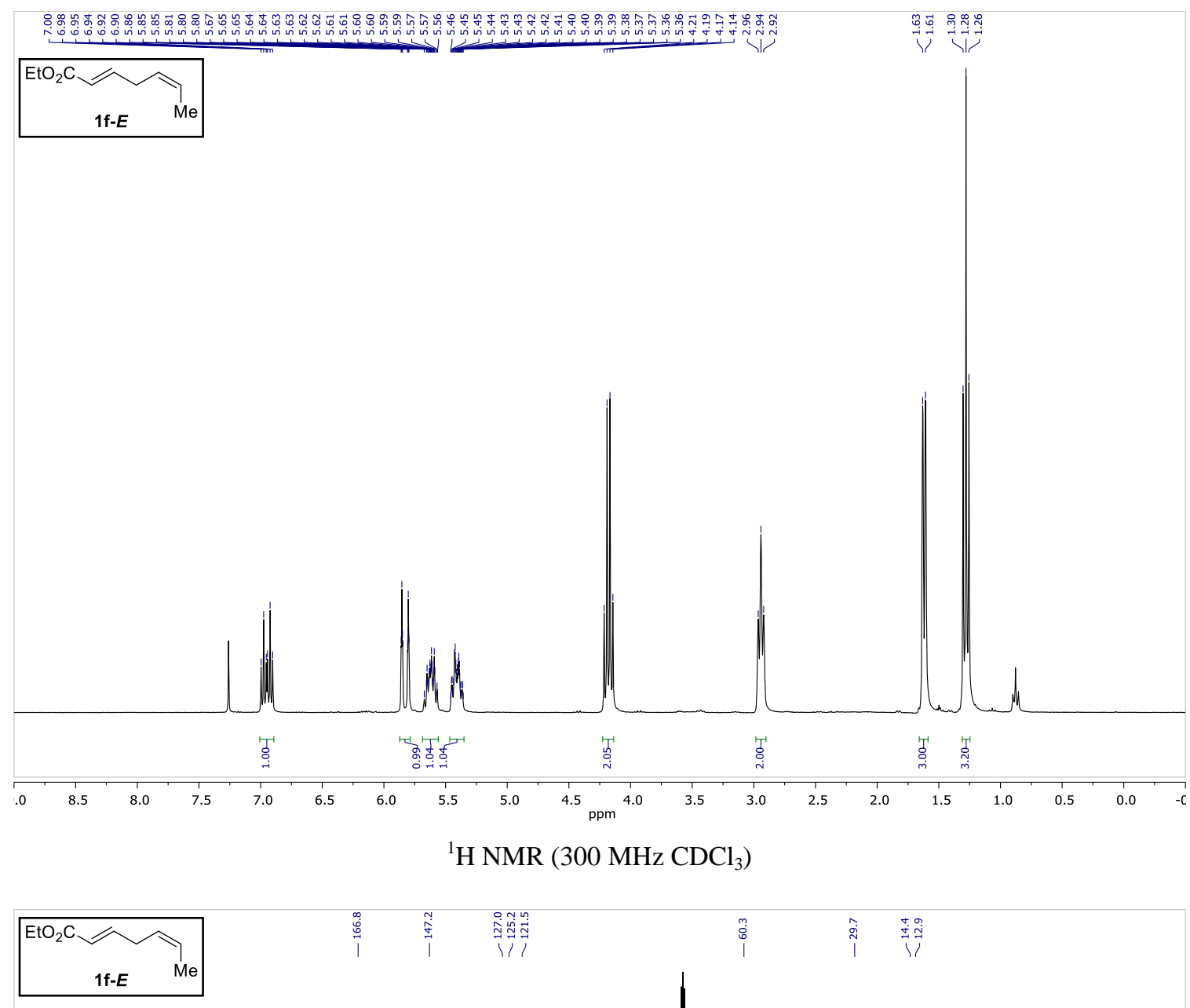

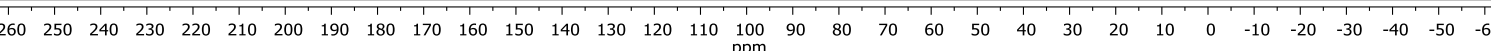

${ }^{13} \mathrm{C}\left\{{ }^{1} \mathrm{H}\right\} \mathrm{NMR}\left(75 \mathrm{MHz}, \mathrm{CDCl}_{3}\right)$ 
Ethyl (2E,5Z)-5-methylhepta-2,5-dienoate (1g)

$\mathrm{EtO}_{2} \mathrm{C}$

تصأ

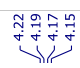

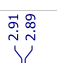

疍

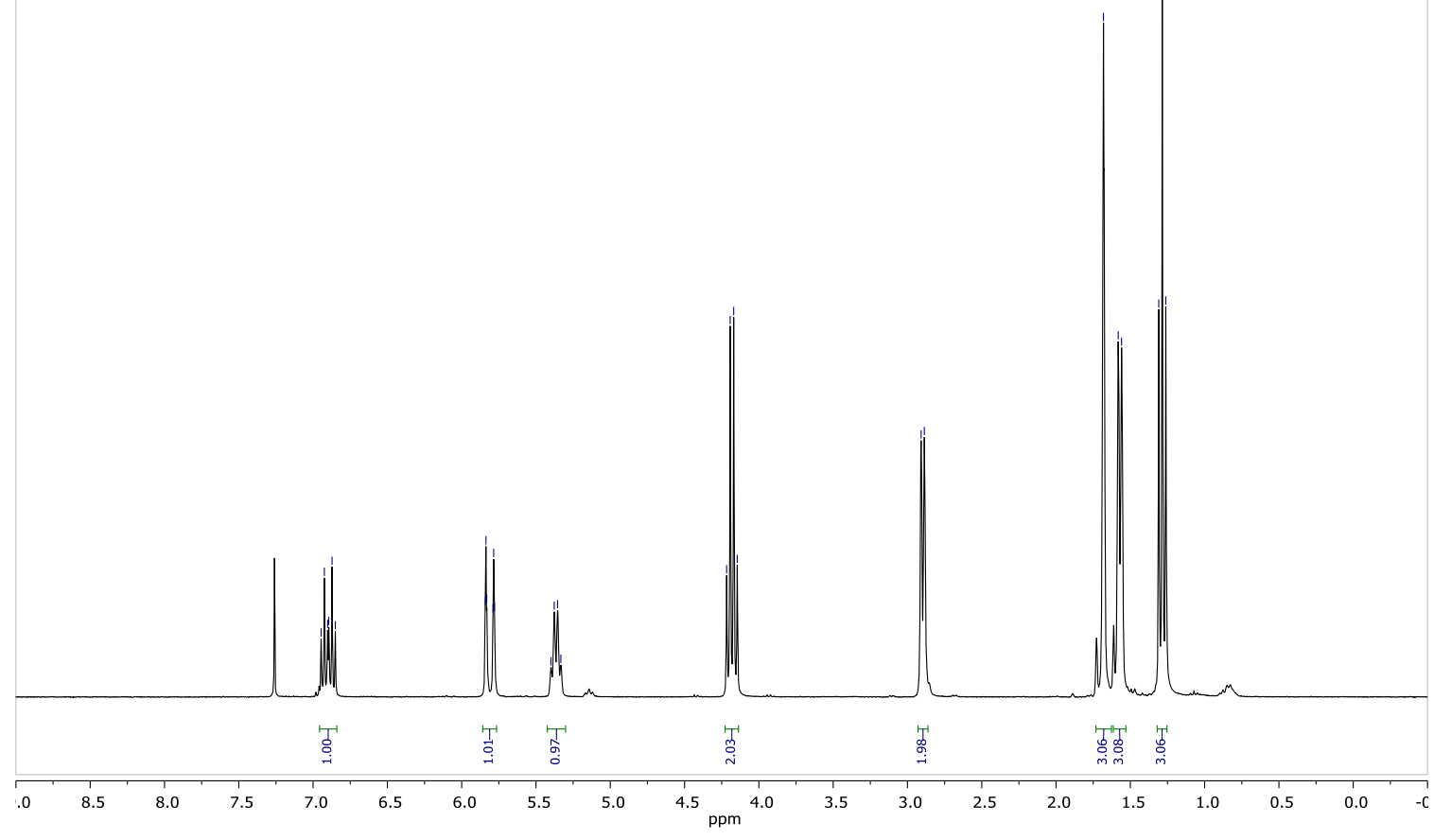

${ }^{1} \mathrm{H}$ NMR (300 MHz $\mathrm{CDCl}_{3}$ )

$\mathrm{EtO}_{2} \mathrm{C}$


Ethyl (2E,4E)-6-hydroxy-5,6-dimethylhepta-2,4-dienoate (4e)

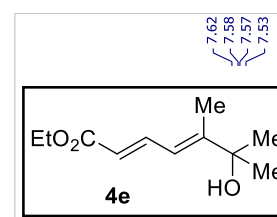

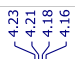

ปั่
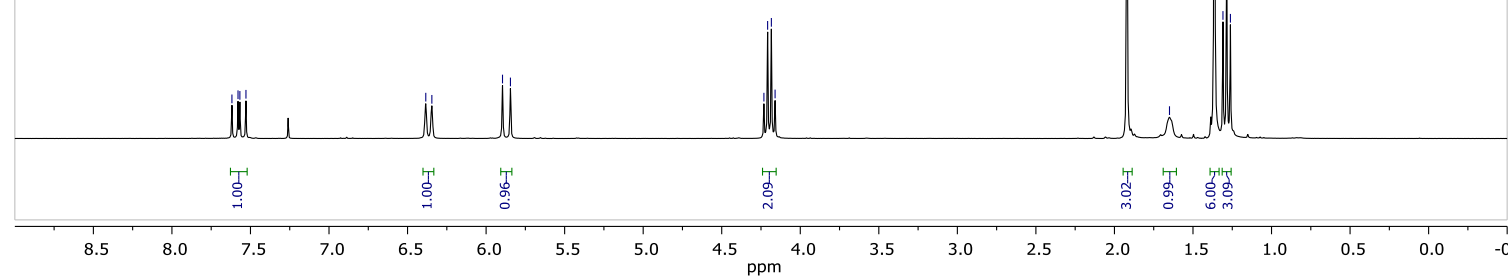

${ }^{1} \mathrm{H}$ NMR $\left(300 \mathrm{MHz} \mathrm{CDCl}_{3}\right)$
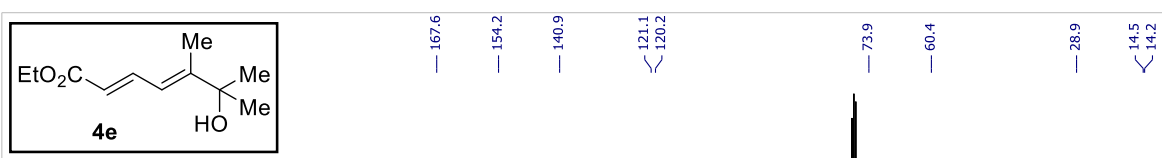

$\begin{array}{llllllllllllllllllllllllllllllllllllllllllll}260 & 250 & 240 & 230 & 220 & 210 & 200 & 190 & 180 & 170 & 160 & 150 & 140 & 130 & 120 & 110 & 100 & 90 & 80 & 70 & 60 & 50 & 40 & 30 & 20 & 10 & 0 & -10 & -20 & -30 & -40 & -50 & -6\end{array}$

${ }^{13} \mathrm{C}\left\{{ }^{1} \mathrm{H}\right\}$ NMR $\left(75 \mathrm{MHz}, \mathrm{CDCl}_{3}\right)$ 
Ethyl (2E,4E)-6-hydroxyhepta-2,4-dienoate (4f)

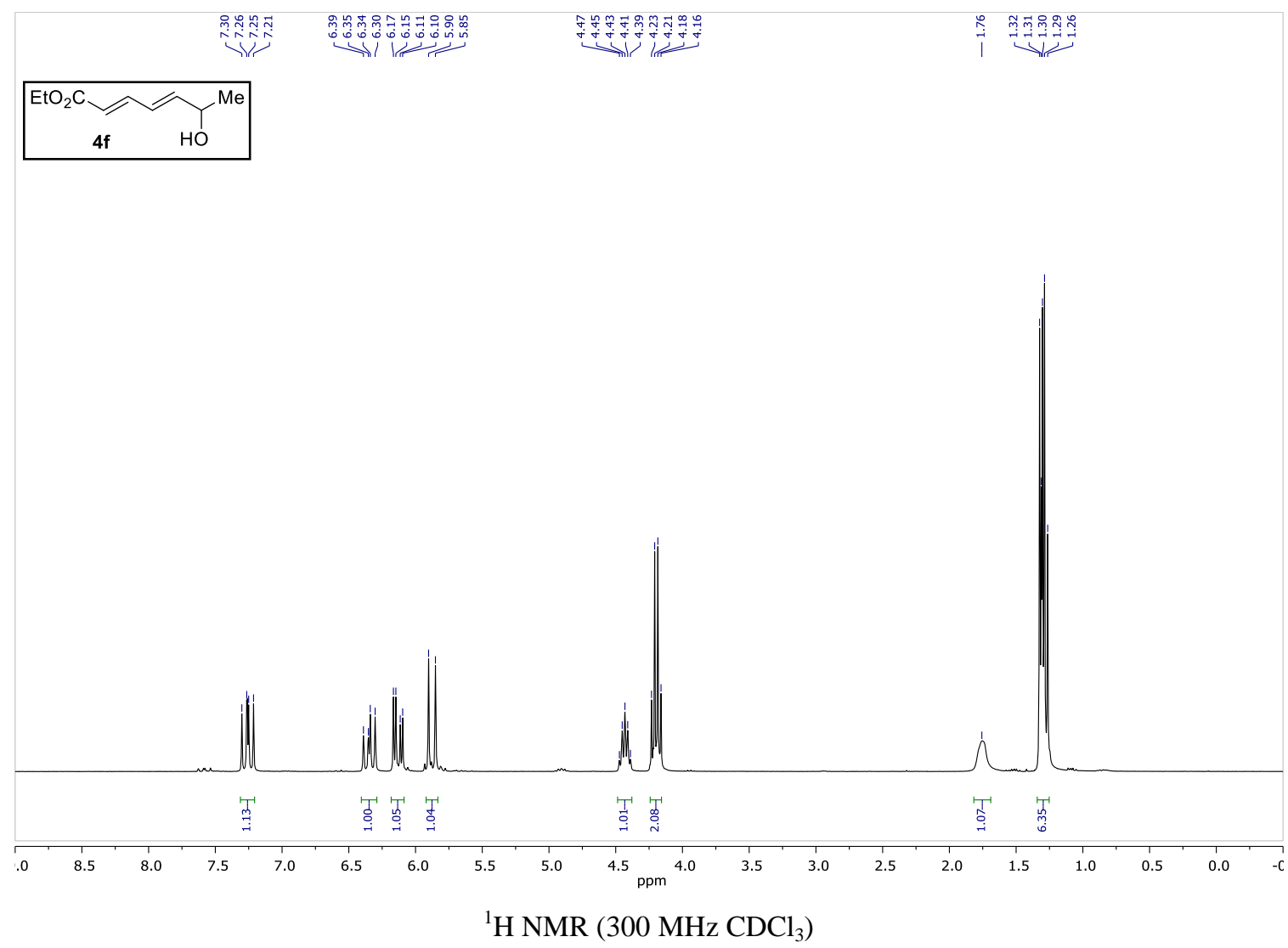

\begin{tabular}{|c|c|c|c|c|c|}
\hline $\mathrm{HO}_{\mathrm{HO}}^{\mathrm{Me}}$ & $\begin{array}{c}\overrightarrow{+} \\
\stackrel{0}{0} \\
1\end{array}$ & 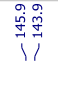 & 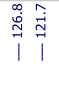 & $\begin{array}{cc}\overrightarrow{0} & n \\
0 & 0 \\
0 & 0 \\
1 & 1\end{array}$ & $\stackrel{\sim}{\tilde{N}}$ \\
\hline
\end{tabular}

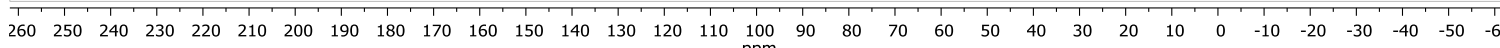

${ }^{13} \mathrm{C}\left\{{ }^{1} \mathrm{H}\right\}$ NMR $\left(75 \mathrm{MHz}, \mathrm{CDCl}_{3}\right)$ 
Ethyl (2E,4E)-6-hydroxy-5-methylhepta-2,4-dienoate (4g)
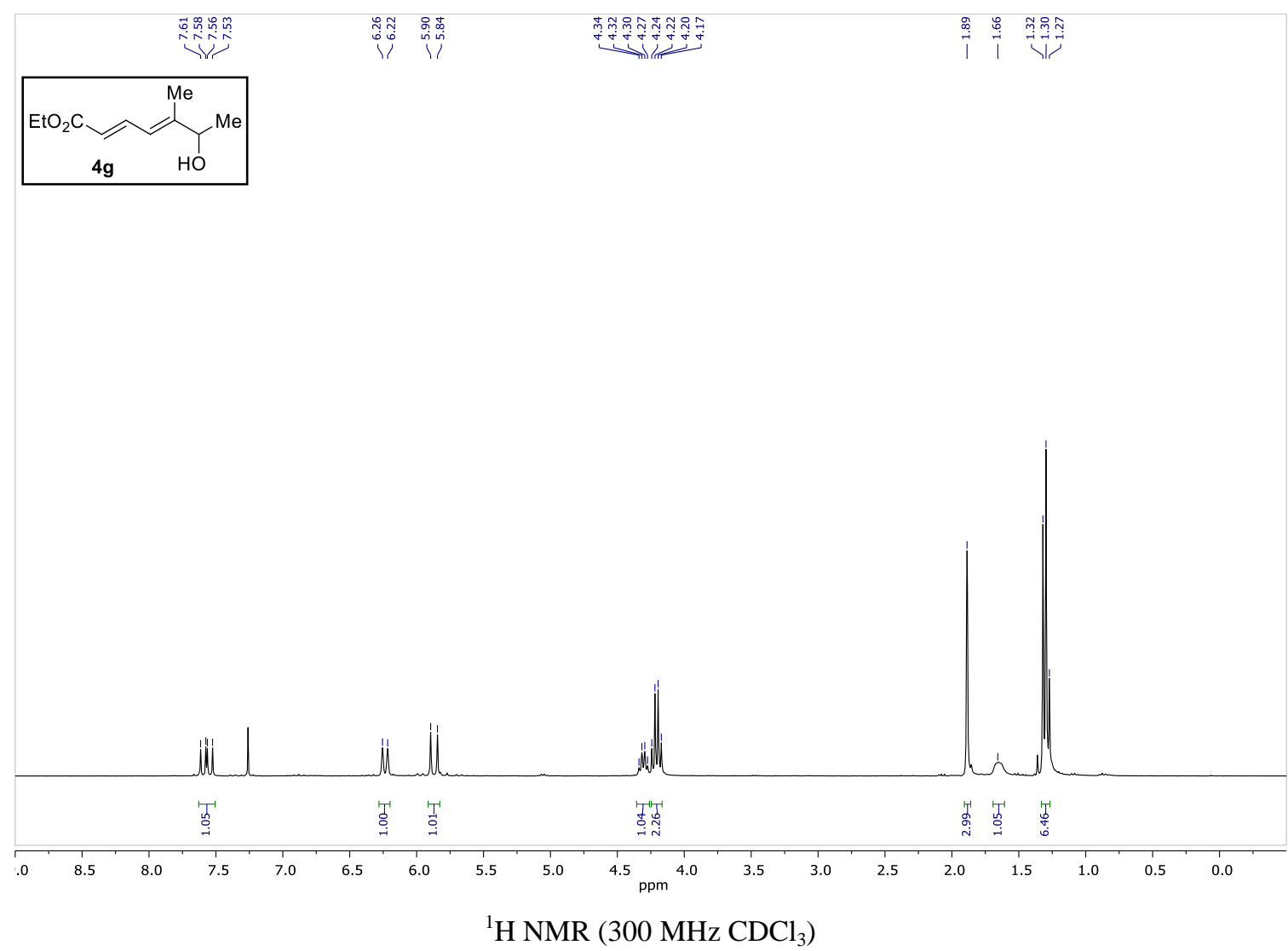

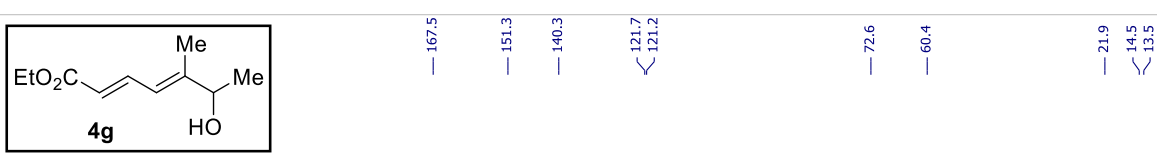

$\begin{array}{llllllllllllllllllllllllllllllllll}260 & 250 & 240 & 230 & 220 & 210 & 200 & 190 & 180 & 170 & 160 & 150 & 140 & 130 & 120 & 110 & 100 & 90 & 80 & 70 & 60 & 50 & 40 & 30 & 20 & 10 & 0 & -10 & -20 & -30 & -40 & -50 & -6\end{array}$

${ }^{13} \mathrm{C}\left\{{ }^{1} \mathrm{H}\right\} \mathrm{NMR}\left(75 \mathrm{MHz}, \mathrm{CDCl}_{3}\right)$ 
Ethyl (2E,4E)-6-methoxy-5,6-dimethylhepta-2,4-dienoate (5)
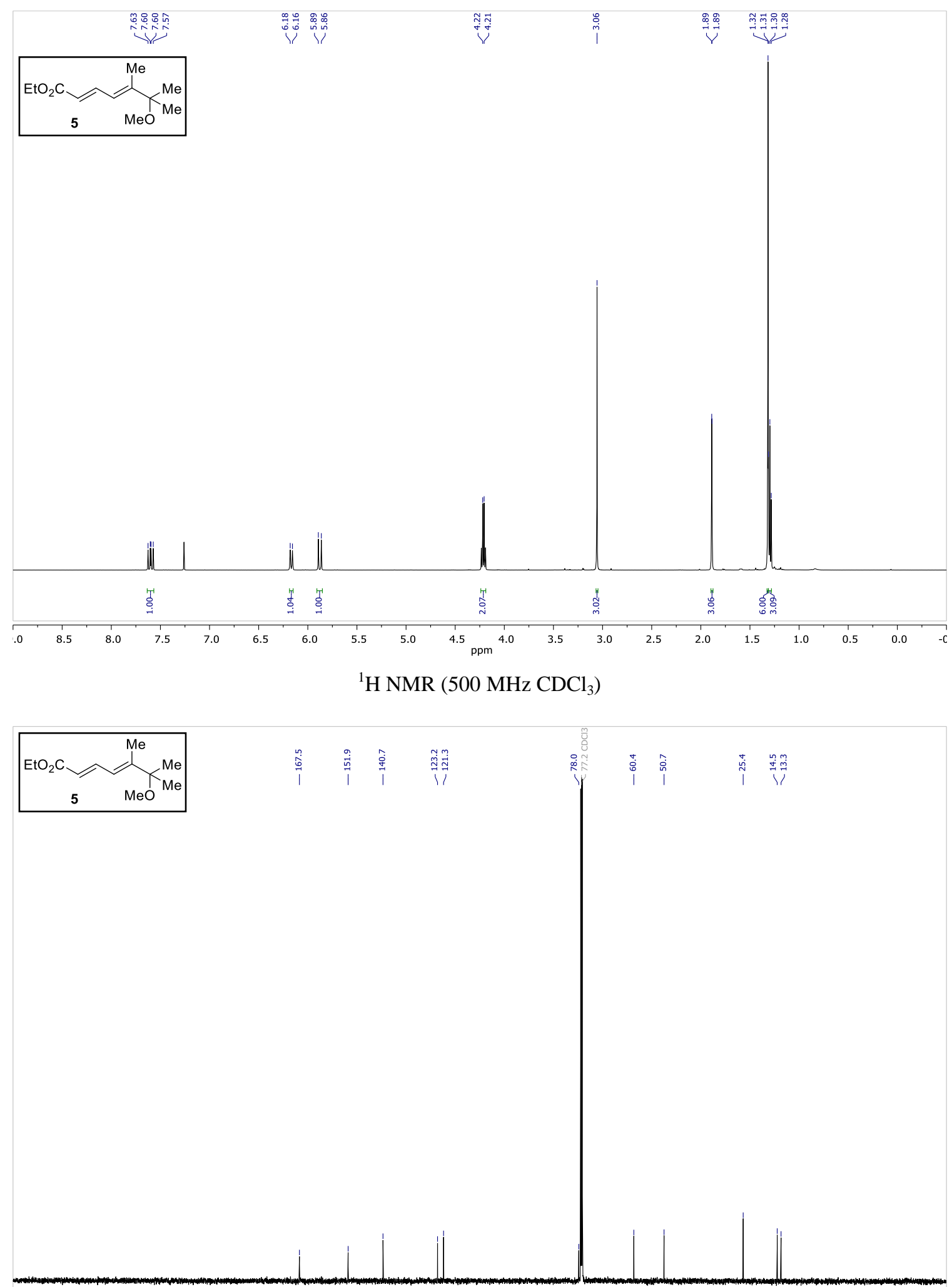

$\begin{array}{llllllllllllllllllllllllllllllllllll}250 & 240 & 230 & 220 & 210 & 200 & 190 & 180 & 170 & 160 & 150 & 140 & 130 & 120 & 110 & 100 & 90 & 80 & 70 & 60 & 50 & 40 & 30 & 20 & 10 & 0 & -10 & -20 & -30\end{array}$

${ }^{13} \mathrm{C}\left\{{ }^{1} \mathrm{H}\right\} \mathrm{NMR}\left(126 \mathrm{MHz}, \mathrm{CDCl}_{3}\right)$ 
Ethyl (2E,4E)-6-ethoxy-5,6-dimethylhepta-2,4-dienoate (6)

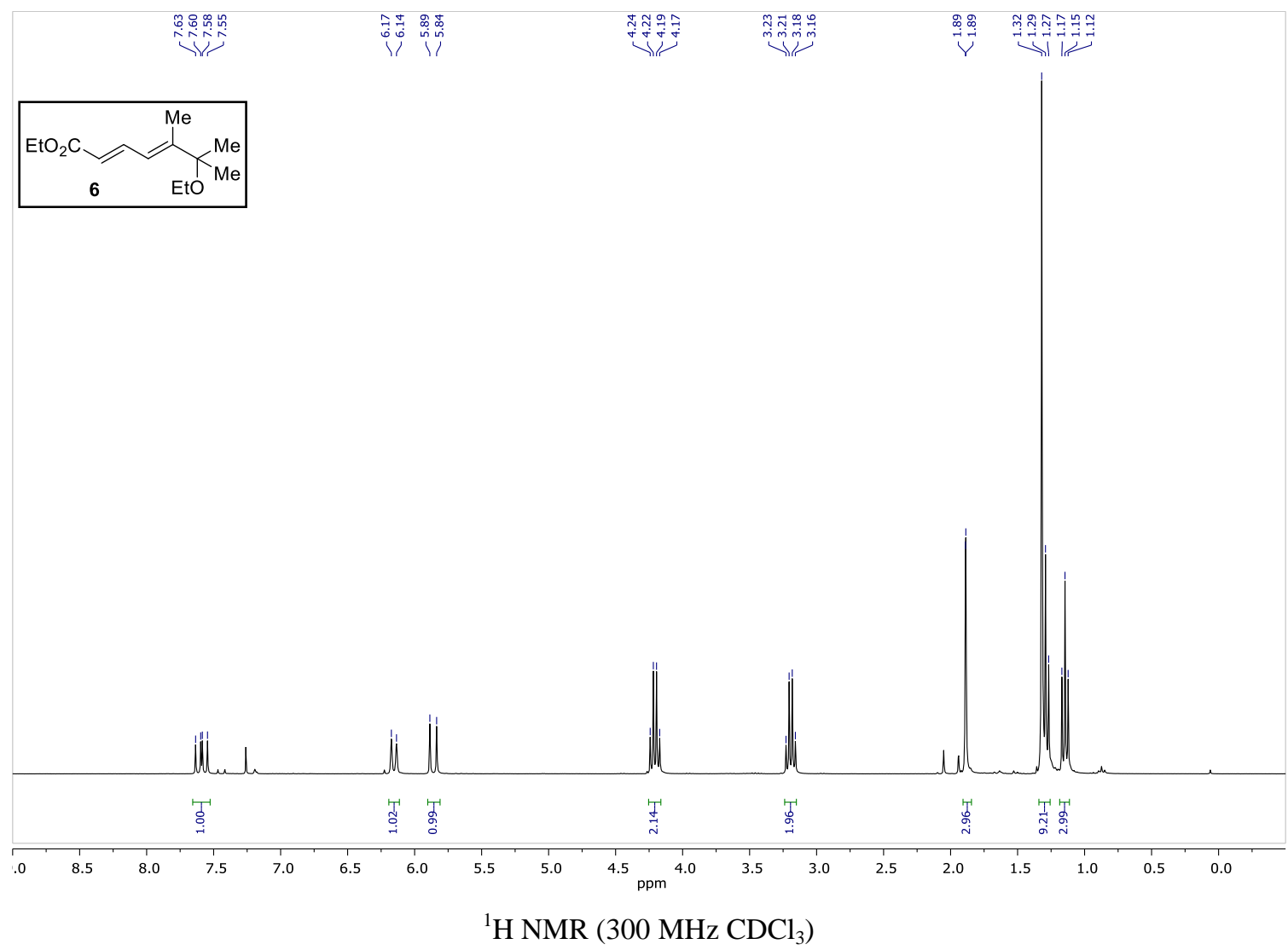

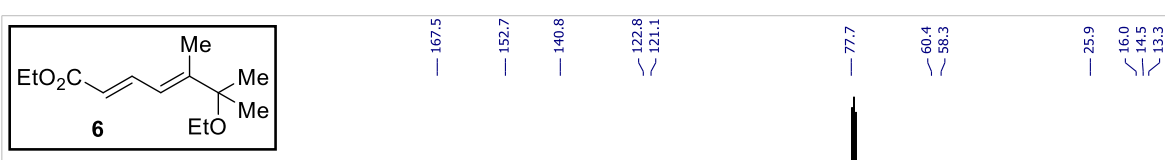


Ethyl (2E,4E)-5,6-dimethylhepta-2,4,6-trienoate (7)
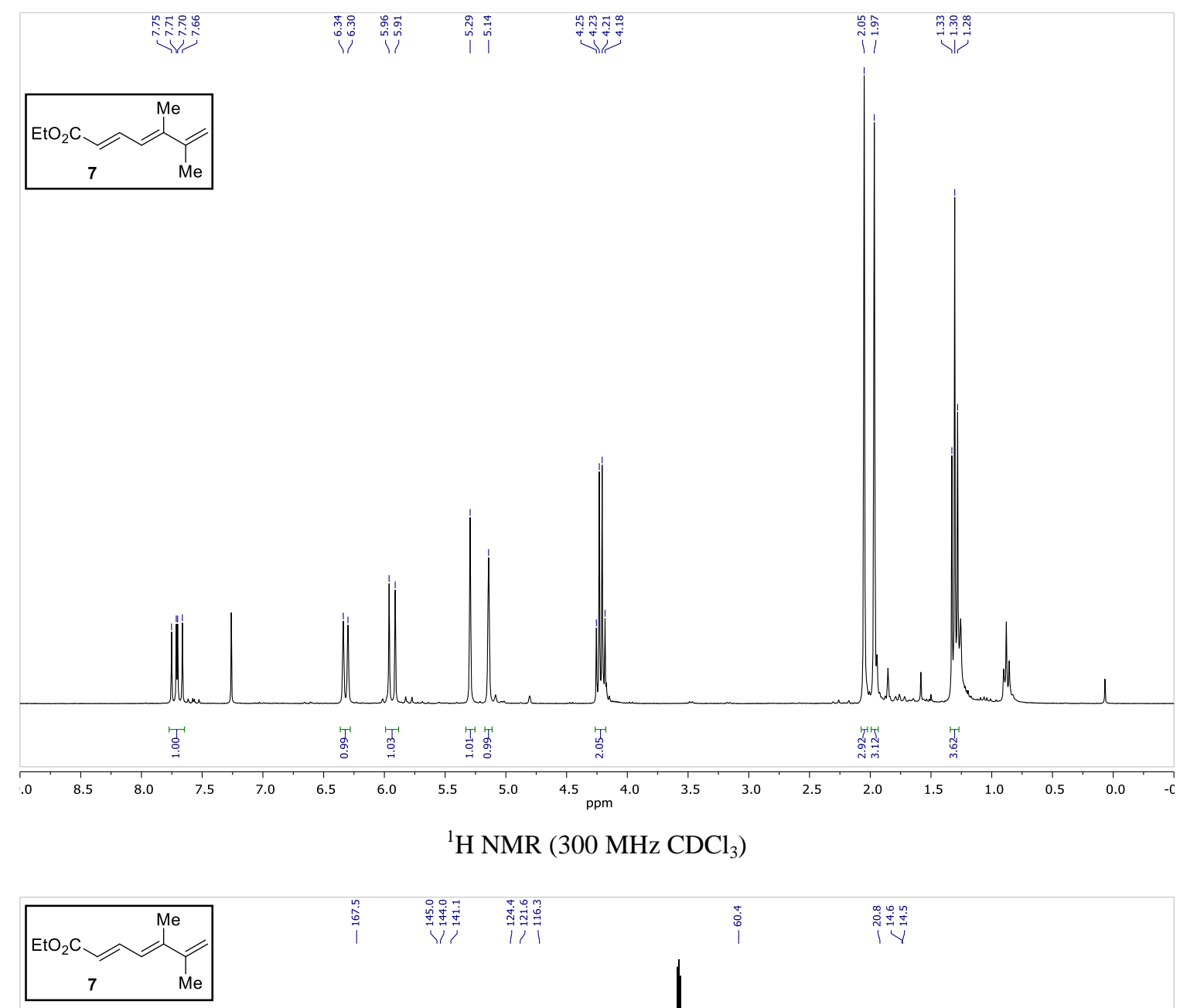

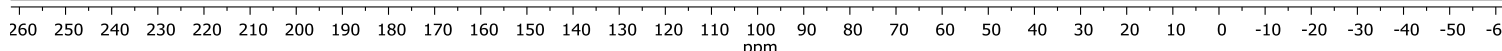

${ }^{13} \mathrm{C}\left\{{ }^{1} \mathrm{H}\right\}$ NMR $\left(75 \mathrm{MHz}, \mathrm{CDCl}_{3}\right)$ 
Ethyl (2E,4E)-6-methoxyhepta-2,4-dienoate (8)
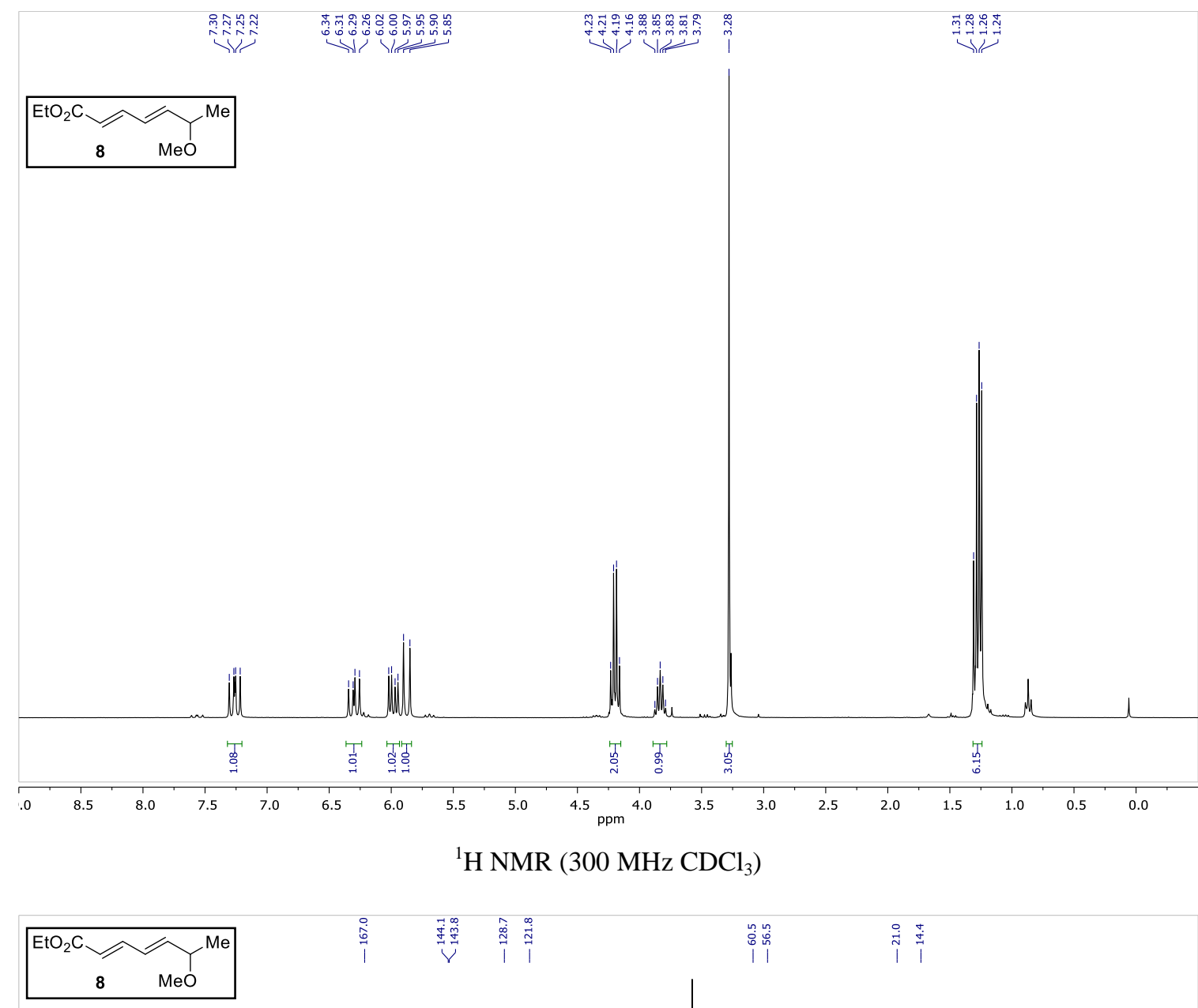

$\begin{array}{llllllllllllllllllllllllllllllllllllllll} & 260 & 250 & 240 & 230 & 220 & 210 & 200 & 190 & 180 & 170 & 160 & 150 & 140 & 130 & 120 & 110 & 100 & 90 & 80 & 70 & 60 & 50 & 40 & 30 & 20 & 10 & 0 & -10 & -20 & -30 & -40 & -50 & -6\end{array}$

${ }^{13} \mathrm{C}\left\{{ }^{1} \mathrm{H}\right\}$ NMR $\left(75 \mathrm{MHz}, \mathrm{CDCl}_{3}\right)$ 


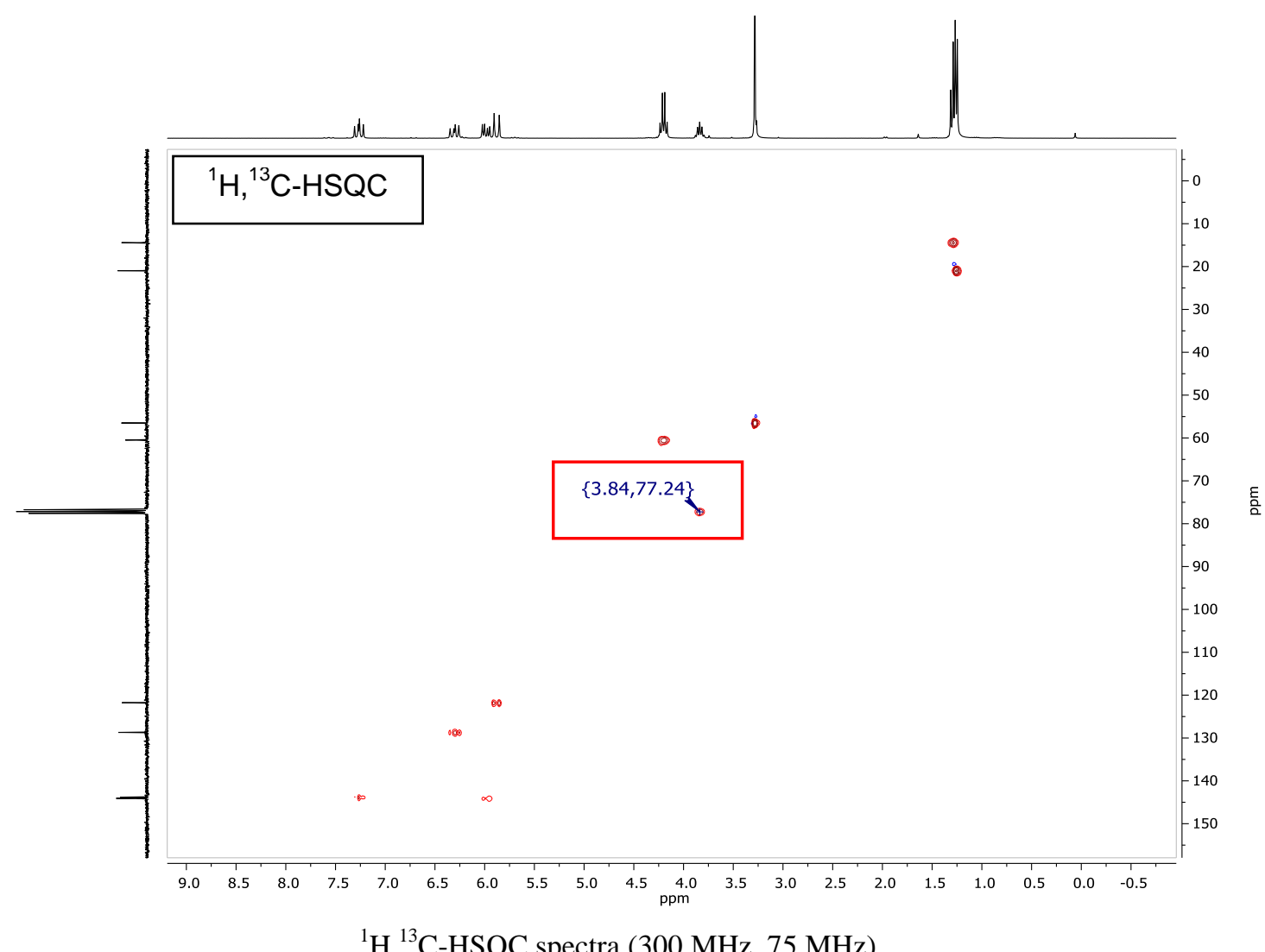

${ }^{1} \mathrm{H},{ }^{13} \mathrm{C}-\mathrm{HSQC}$ spectra $(300 \mathrm{MHz}, 75 \mathrm{MHz})$ 
Ethyl (2E,5Z)-5-methyl-4-oxohepta-2,5-dienoate (9)
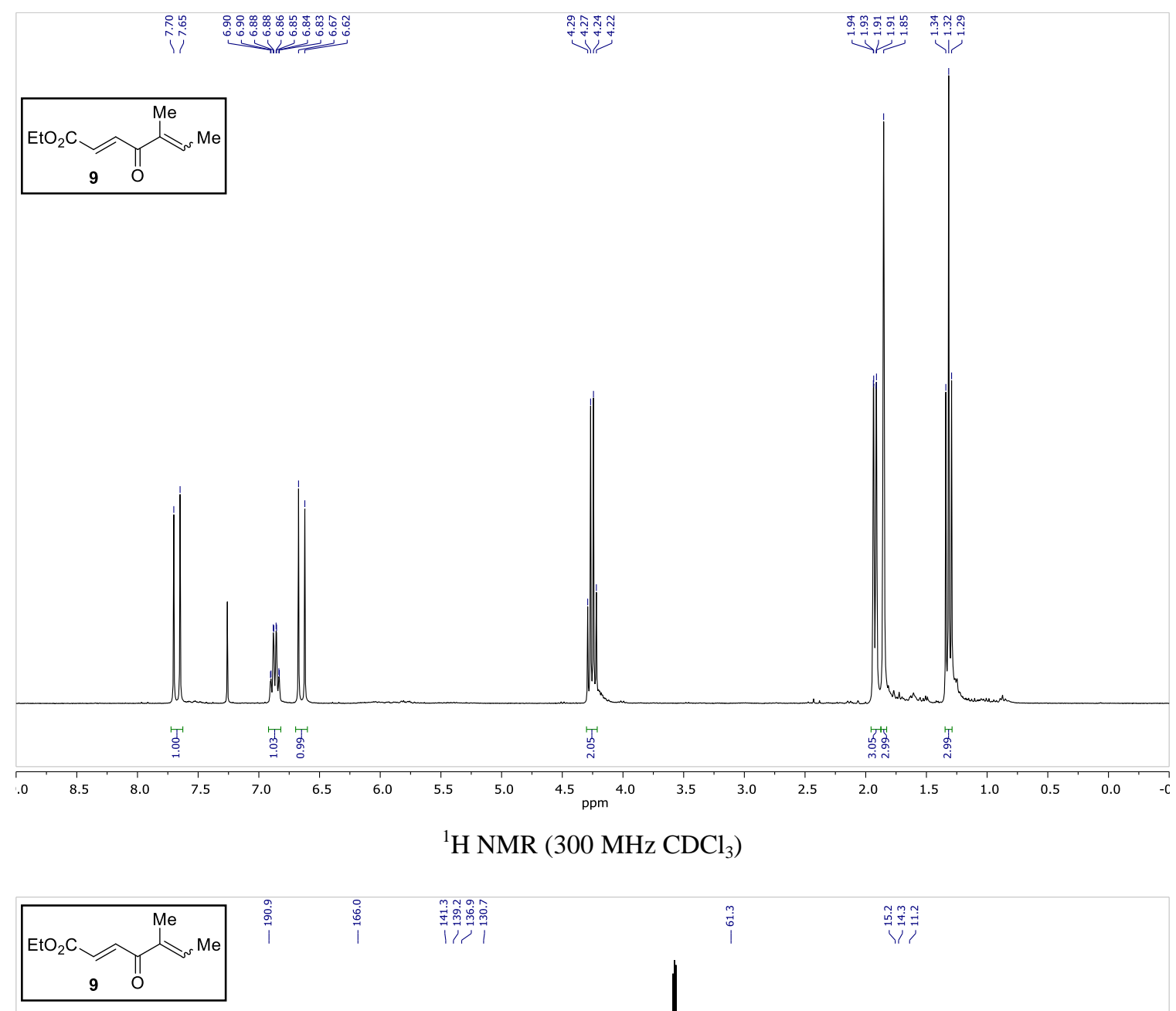

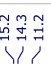

$\begin{array}{llllllllllllllllllllllllllllllllll}260 & 250 & 240 & 230 & 220 & 210 & 200 & 190 & 180 & 170 & 160 & 150 & 140 & 130 & 120 & 110 & 100 & 90 & 80 & 70 & 60 & 50 & 40 & 30 & 20 & 10 & 0 & -10 & -20 & -30 & -40 & -50 & -6\end{array}$

${ }^{13} \mathrm{C}\left\{{ }^{1} \mathrm{H}\right\}$ NMR $\left(75 \mathrm{MHz}, \mathrm{CDCl}_{3}\right)$ 
Ethyl (2E,4E)-6-methoxy-5-methylhepta-2,4-dienoate (10)

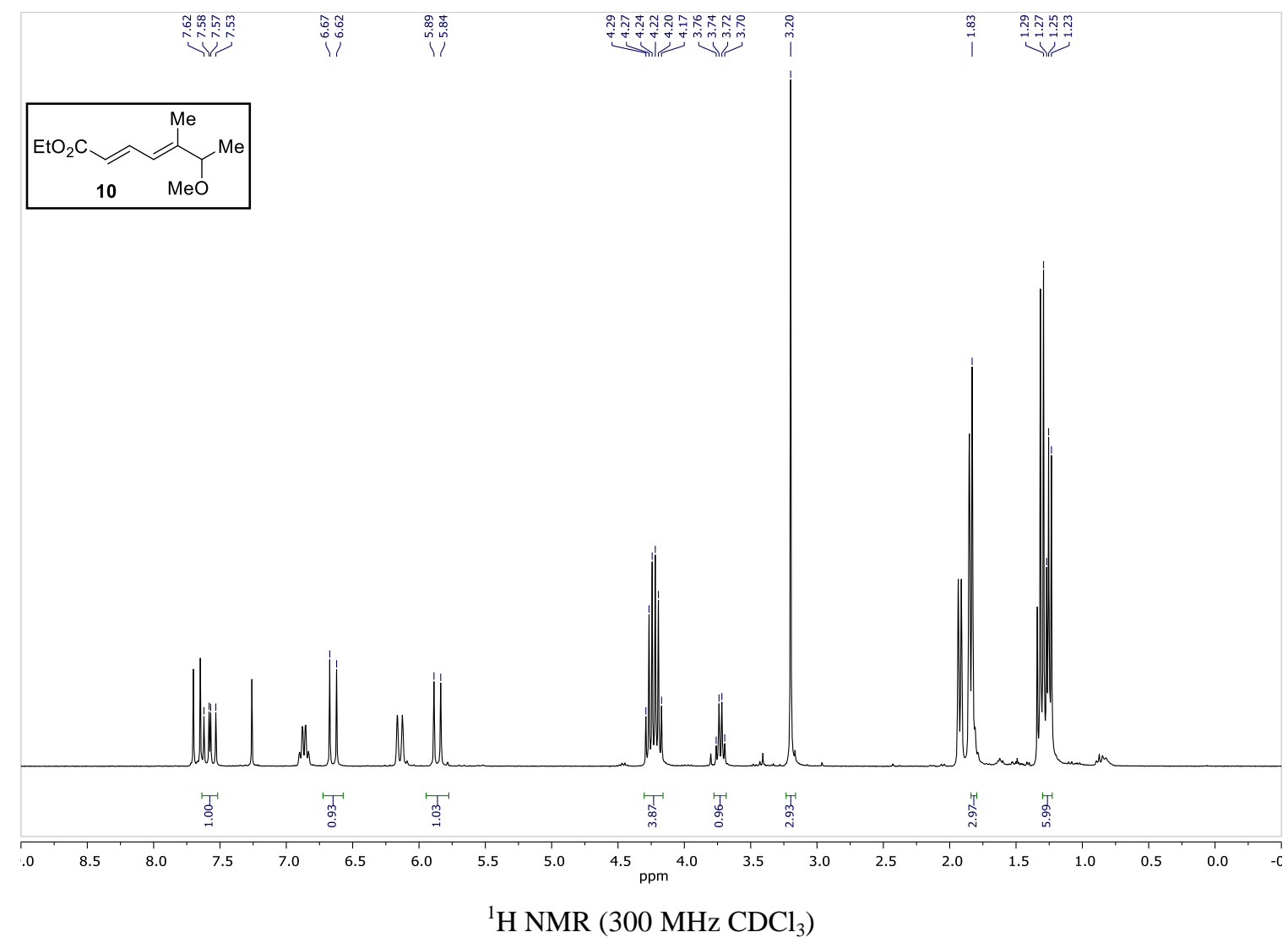

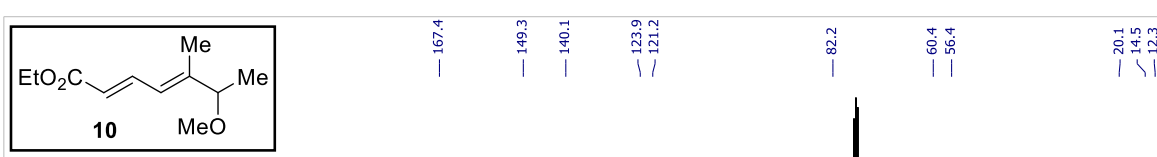

$\begin{array}{llllllllllllllllllllllllllllllllll}260 & 250 & 240 & 230 & 220 & 210 & 200 & 190 & 180 & 170 & 160 & 150 & 140 & 130 & 120 & 110 & 100 & 90 & 80 & 70 & 60 & 50 & 40 & 30 & 20 & 10 & 0 & -10 & -20 & -30 & -40 & -50 & -6\end{array}$

${ }^{13} \mathrm{C}\left\{{ }^{1} \mathrm{H}\right\} \mathrm{NMR}\left(75 \mathrm{MHz}, \mathrm{CDCl}_{3}\right)$ 
Ethyl (2E,5E)-3-methyl-4-oxo-6-(trimethylsilyl)hexa-2,5-dienoate (11)
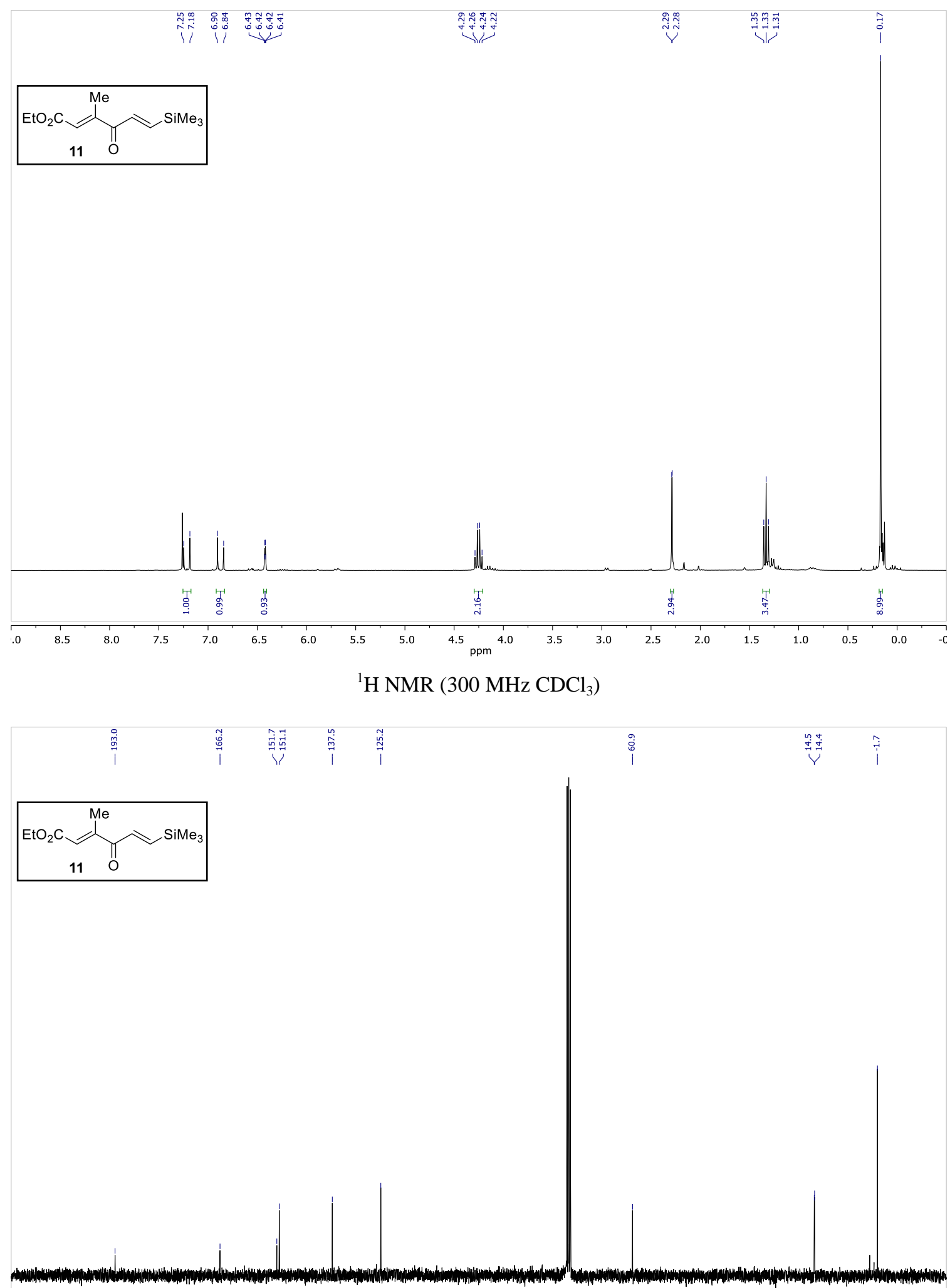

$\begin{array}{lllllllllllllllllllllll}210 & 200 & 190 & 180 & 170 & 160 & 150 & 140 & 130 & 120 & 110 & 100 & 90 & 80 & 70 & 60 & 50 & 40 & 30 & 20 & 10 & 0 & -10\end{array}$

${ }^{13} \mathrm{C}\left\{{ }^{1} \mathrm{H}\right\}$ NMR $\left(75 \mathrm{MHz}, \mathrm{CDCl}_{3}\right)$ 
${ }^{77} \mathrm{Se}$ NMR spectra of $\mathrm{SeO}_{2}$ in $\mathrm{CD}_{3} \mathrm{CN} / \mathrm{D}_{2} \mathrm{O} 1: 1(\mathrm{v} / \mathrm{v})$

$$
\begin{aligned}
& { }^{77} \mathrm{Se} \mathrm{NMR}(95.5 \mathrm{MHz}, 305 \mathrm{~K}) \\
& \mathrm{SeO}_{2} \text { in } \mathrm{CD}_{3} \mathrm{CN} / \mathrm{D}_{2} \mathrm{O} 1: 1(\mathrm{v} / \mathrm{v})
\end{aligned}
$$

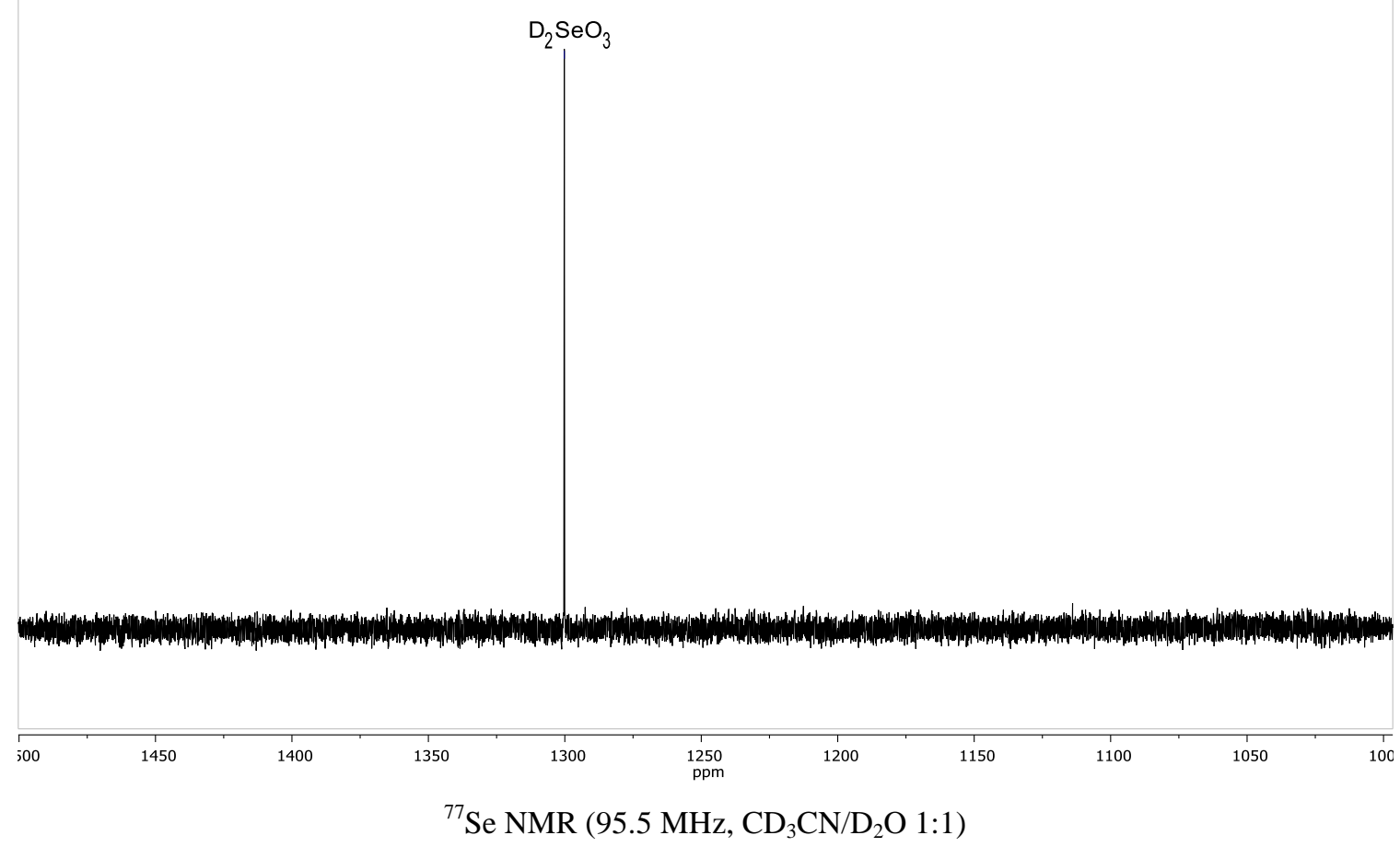

The observed signal at $1300 \mathrm{ppm}$ for $\mathrm{D}_{2} \mathrm{SeO}_{3}$ in ${ }^{77} \mathrm{Se}$ NMR is in accordance with the literature from Milne. ${ }^{1}$ 
GC-FID and GC/MS spectra for entry 3 in Table 1 (reaction optimization)

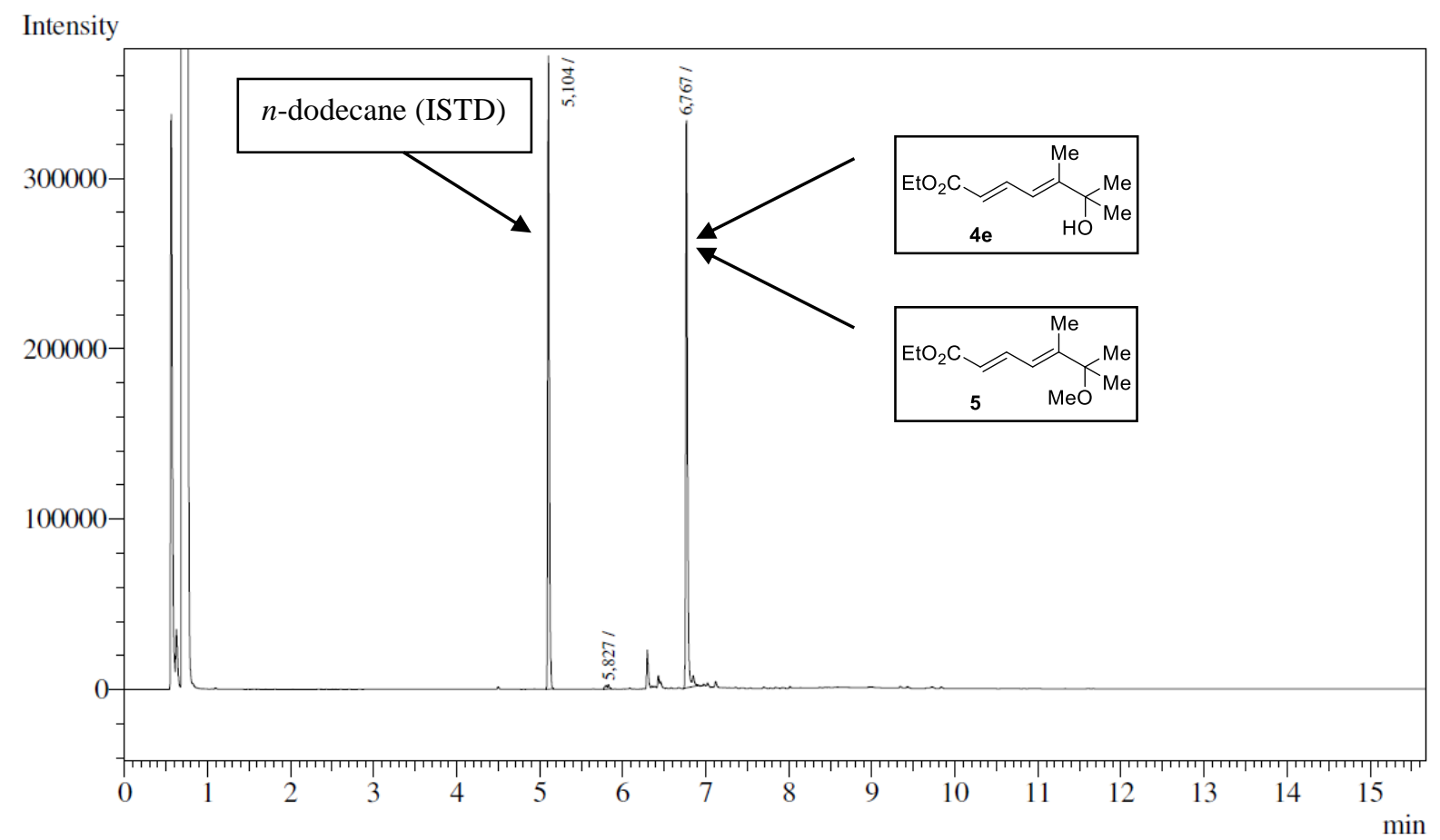

\begin{tabular}{rrrrrrr}
\multicolumn{1}{c}{ Peak\# } & Ret.Time & \multicolumn{1}{c}{ Area } & Height & \multicolumn{1}{c}{ Conc. } & Unit Mark ID\# & Cmpd Name \\
1 & 5,104 & 471477 & 364912 & 50,019 & & \\
2 & 5,827 & 7156 & 2508 & 0,759 & & \\
3 & 6,767 & 463965 & 314314 & 49,222 & & \\
\hline Total & 942598 & 681734 & &
\end{tabular}

Figure 1: GC-FID chromatogram after 60 min reaction time.

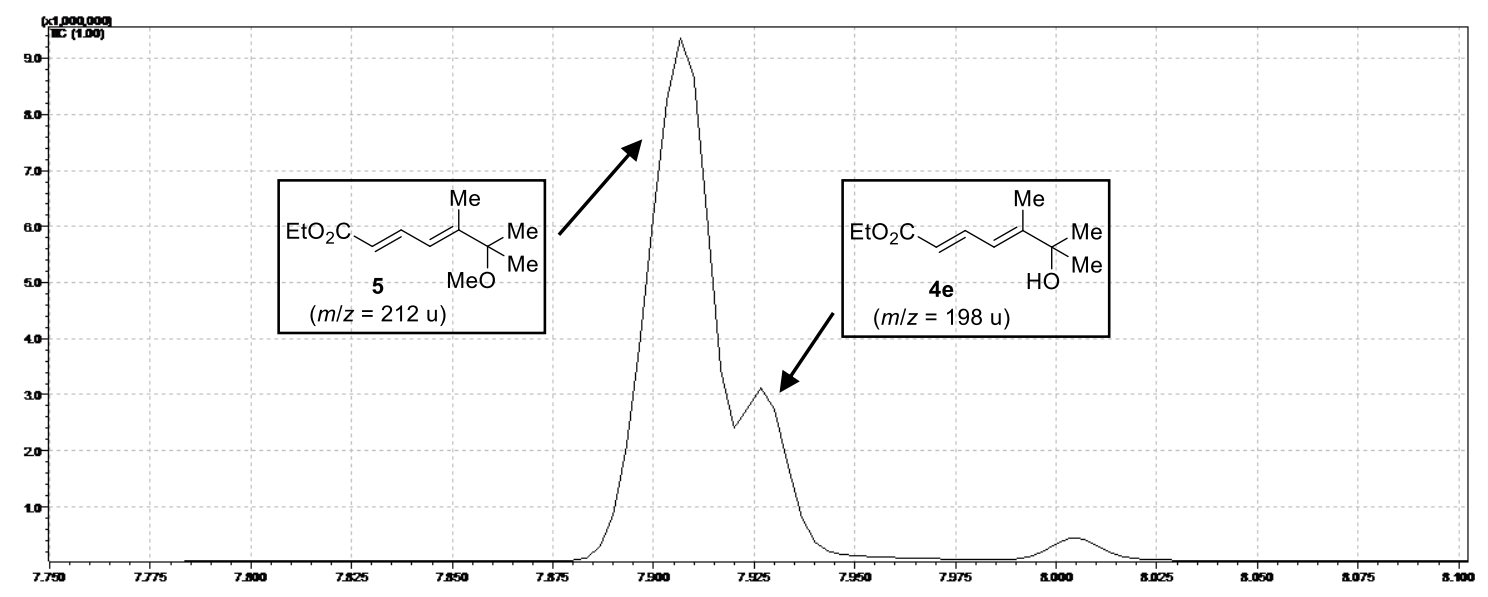

Figure 2: Total ion current chromatogram of GC/MS analysis.

\section{References}

(1) Milne, J. Magn. Reson. Chem. 1993, 31, 652-655. 